\title{
Tea consumption and the reduced risk of colon cancer - results from a national prospective cohort study
}

\author{
L Joseph $\mathrm{Su}^{1, *}$ and Lenore Arab² \\ 'Stanley S Scott Cancer Center and Departments of Public Health and Preventive Medicine, \\ Louisiana State University Health Sciences Center, 1600 Canal Street, Suite 800, New Orleans, LA 70112, USA: \\ ${ }^{2}$ Departments of Epidemiology and Nutrition, University of North Carolina, Chapel Hill, NC 27599, USA
}

Submitted 26 January 2001: Accepted 19 0ctober 2001

\begin{abstract}
Objective: This study examines the relationship between tea consumption and colon cancer risk in the US population.

Design: Data from the NHANES I Epidemiologic Follow-up study (NHEFS) were used to examine the hypothesis. Cox proportional hazard models were used to examine the hypothesis of a protective effect of frequent tea consumption on colon cancer occurrence.

Setting: Due to differences in the precision of the exposure data, we analysed two cohort periods based on the NHEFS. Cohort I was based on the survey conducted at the NHEFS baseline and Cohort II began at the first follow-up.

Subjects: After excluding non-incidence cases and cases lost to follow-ups, there were 2359 tea users and 6498 non-tea users at baseline and 7656 tea users and 4514 non-tea users at the first follow-up.

Results: After adjusting for confounders, the relative risks of colon cancer are 0.57 (95\% confidence interval (CI) $0.42,0.78)$ and 0.59 (95\% CI $=0.35,1.00)$ for subjects who consumed $\leq 1.5$ cups and $>1.5$ cups per day, respectively, compared with nontea users in Cohort II. Although more women consumed tea and the mean intake was higher, the preventive effect of tea consumption on colon cancer was found predominantly in men. The relative risks of colon cancer are $0.41(95 \% \mathrm{CI}=0.25$, $0.66)$ for men who consumed $\leq 1.5 \mathrm{cups}^{-1} \mathrm{day}^{-1}$ and $0.30(95 \% \mathrm{CI}=0.09,0.98)$ for $>1.5$ cups day $^{-1}$ of tea consumption $(P$-value for trend $<0.01)$. No significant results were found in Cohort I.

Conclusions: This study suggests an inverse association between colon cancer risk and habitual tea consumption.
\end{abstract}

Colon cancer is the second leading cause of cancer death in the United States ${ }^{1-3}$. The American Cancer Society estimates that about 93800 new cases of colon cancer will be diagnosed in the year 2000 and account for about $8 \%$ of all newly diagnosed cancers ${ }^{3}$. The identification of risk factors for colon cancer and subsequent intervention against the risk factors, especially in the at-risk population, hold greater promise for achieving the goal of decreasing mortality rates from this disease than does treatment.

Although a genetic component is well established, 85\% of colon cancer cases are still considered 'sporadic'. In addition, indirect evidence, based largely on the differences in cancer rates across countries ${ }^{4}$ and changes in rates over time and among migrants $5^{5-7}$, implies that environmental factors - diet in particular - are also important. A search for the dietary risk factors for colon cancer is warranted in order to reduce colon cancer incidence and the healthcare costs of this disease.
Tea has a history of human use of over 4000 years and is the second most commonly consumed beverage in the world. There are emerging data on the anti-carcinogenic benefits of tea consumption. In vitro experiments and in animal models have shown tea to be an effective agent against chemically induced tumorigenesis, affecting induction, tumour size and metastases ${ }^{8-11}$. Epidemiological study results, however, have been inconsistent in showing the beneficial effect of tea consumption on cancer prevention.

The First National Health and Nutrition Examination Survey (NHANES I) Epidemiologic Follow-up Study (NHEFS) is a prospective cohort study with a probability sample of men and women from the non-institutionalised US population and includes a wide range in age, socioeconomic status and ethnicity. This study uses the NHEFS data to examine whether there is an inverse association between habitual tea consumption and colon cancer risk over an approximately 20 -year follow-up period. 


\section{Methods}

\section{Study population}

Detailed descriptions of NHANES I and NHEFS have been published previously ${ }^{12-18}$. In brief, NHANES I was conducted by the National Center for Health Statistics from 1971 to 1975. Baseline data on sociodemographic characteristics, medical history, diet, and biochemical and anthropometric measures were collected from a US national probability sample of non-institutionalised persons aged 25 to 74 years. Population subgroups thought to be at 'nutritional risk' were over-sampled. Four waves of follow-up for the 14407 subjects who underwent the medical examination were conducted in the NHEFS during 1982-84, 1986, 1987 and 1992. Those who had died were followed up by proxy interview. Of the 14407 participants eligible for follow-up, 13291 (92.2\%) were successfully traced through 1992 . Death certificates were coded according to the ninth revision of the International Classification of Diseases (ICD-9).

\section{Colon cancer cases}

Cancer morbidity was self-reported in four follow-up interviews; cancer mortality was ascertained through proxy interview and information from the National Death Index. In rare situations where the year of first cancer diagnosis was not available, the midpoint between two interviews was used as the year of cancer incidence. The cause of death was validated by referring to the primary cause of death listed on the death certificate by the NCHS. International Classification of Diseases ICD-9-CM code 153 was used to identify colon cancer morbidity as the first cancer incidence other than skin cancer. Cancer cases that reported having had other cancers previously were excluded from the analysis. If subjects reported multiple cancers in the same year and colon cancer was among the cancers, colon cancer was selected as the primary cancer incidence. Overall, colon cancer cases were determined primarily from the morbidity information. If subjects died of colon cancer but never reported colon cancer morbidity, the subjects were assigned as colon cancer cases and the time of death was identified as the cancer incidence. The follow-up time of cases was calculated by subtracting the month and year of the baseline interview from the mid-year of the 'incidence' of colon cancer. Similarly, for lost subjects, the follow-up time was estimated as the total number of months between baseline interview and the time of censoring. The censoring time was either the time of dropout (loss to follow-up or cancer of other sites) or the end of the study in July 1993.

\section{Tea consumption}

Tea consumption status was based on a 24-hour dietary recall data and one question on consumption of tea and coffee in the baseline survey (1972-74) and the food-frequency questionnaire solicited in the 1982-84 follow-up survey. The baseline questionnaire did not distinguish between tea and coffee consumption. Therefore, if subjects reported tea drinking in the 24-hour dietary recall regardless of the response to the single question in the questionnaire, the amount of tea consumption is used as daily tea intake. However, if subjects responded 'yes' to the question but reported no coffee consumption in the 24-hour recall, the average amount of tea consumed by tea users in the NHEFS is used to define the amount of tea consumption. The foodfrequency questionnaire conducted in 1982-84 contained much more detail about tea drinking practices and frequency of consumption. We are able to directly calculate the amount of daily tea consumption.

\section{Analytic coborts}

Two distinct analytic cohorts were defined. Cohort I was based upon the initial interview with its baseline (1971-75). Cohort II included all persons free of cancer until the first follow-up (1982-84) of the NHEFS. Cohort I was followed for approximately 20 years $(n=14407)$; Cohort II was followed for approximately 10 years $(n=10220)$.

\section{Dietary intakes}

Dietary records were obtained from a single 24-hour recall interview at the baseline survey for 10220 members of the NHEFS cohort. Intakes of 13 nutrients for the foods consumed in the NHANES I baseline survey were calculated by the NCHS. Intakes of total calories, calcium, total fat and dietary fibres were included as covariates to assess possible confounding effects of these on the tea-colon cancer relationship.

\section{Other covariates}

Sociodemographic variables obtained from the baseline survey (age, education, race, gender and poverty index) were included in the examination as possible confounders or effect modifiers. Questions on alcohol consumption were asked at the baselines of both cohorts. Non-drinkers were defined as those who reported no alcohol consumption in the past year. The average number of drinks consumed per week was derived by multiplying the frequency of drinking by the average amount usually consumed. The calculation of body mass index (BMI, $\mathrm{kg} \mathrm{m}^{-2}$ ) was based on self-reported weight and height at baseline. In addition, subjects were asked at the 1982-84 and 1992 interviews whether any close family members (parents/siblings/children) had cancer and, if so, the types of cancer. The information from both waves of interviews was then combined to create the variable for familial colon cancer history. Aspirin use over the 30 days prior to the baseline interview was entered as a dichotomous (yes/no) variable; the use of vitamin or mineral supplements in the past month was also assessed and coded as yes/no. Three 
levels of physical activity were estimated based on the selfreported amount of physical activity engaged in.

\section{Exclusions}

Among the eligible subjects, 172 subjects had either metastasised colon cancer or previously diagnosed cancer before the first follow-up of NHEFS in 1982-84. As a result, 219 colon cancer cases and 9792 non-cases were entered into the study analyses.

\section{Statistical analyses}

Cox proportional hazard models, with adjustment for potential confounders (age, race, gender, education, aspirin use, vitamin/mineral use, body mass index, physical activity, dietary intakes of calories, fat, fibre and calcium, and family history of colon cancer), were used to estimate the relationship between tea consumption and the relative risk of colon cancer. Vitamin and mineral use, aspirin use, alcohol consumption and baseline smoking status were missing for some subjects. Covariate inclusion was based on whether inclusion of a covariate changed the coefficient for the main variable by more than $10 \%$. Family history of colon cancer, vitamin and mineral use, physical activity and smoking at baseline were thus not included in the final models. Both continuous and categorical tea consumption variables were used to model colon cancer risk. Gender and race were assessed for possible effect modification.

\section{Results}

The summary demographic characteristics of the NHEFS Cohorts were stratified by tea consumption status (Table 1). After the exclusion of subjects with incomplete information, this study consisted of 3884 subjects reporting no tea drinking at both baseline (1971-75) and first follow-up (1982-84), 6532 subjects reporting tea consumption at either baseline or first follow-up, and 1919 subjects reporting tea consumption in both surveys. The mean age was significantly higher in non-tea users than in tea users $(P<0.01)$. Men were more likely to be non-tea users than women in this study. A higher percentage (57.9\%) of non-tea users had less education $(<12$ th grade) than users (37.5\% and $34.5 \%$ for short-term and long-term users, respectively). Long-term tea users were more likely to be white, had family history of colon cancer, and take a vitamin/mineral supplement.

Tea consumption at baseline and the risk of colon cancer adjusted for confounders are summarised for the NHEFS Cohort I (Table 2). Although there is generally an inverse relationship between colon cancer risk and whether subjects consume tea or not, the relationship did not achieve statistical significance when the data for men and women were combined or analysed separately. When the association between tea consumption and colon cancer risk was examined based on the amount of tea consumed each day, a non-significant inverse trend

Table 1 Demographic characteristics of the NHEFS population, by tea consumption status*

\begin{tabular}{|c|c|c|c|}
\hline & $\begin{array}{l}\text { Non-tea users } \\
\quad(n=3884)\end{array}$ & $\begin{array}{l}\text { Short-term tea users } \\
\quad(n=6532)\end{array}$ & $\begin{array}{l}\text { Long-term tea users } \\
\qquad(n=1919)\end{array}$ \\
\hline Age (years) & $55.1 \pm 15.1$ & $46.9 \pm 14.8$ & $47.0 \pm 15.0$ \\
\hline BMI $\left(\mathrm{kg} \mathrm{m}^{-2}\right)$ & $25.8 \pm 5.2$ & $25.7 \pm 5.1$ & $25.3 \pm 4.9$ \\
\hline Men & 1957 (50.4\%) & 2436 (37.3\%) & 579 (30.2\%) \\
\hline \multicolumn{4}{|l|}{ Colon cancer case $†$} \\
\hline Yes & $112(2.9 \%)$ & $110(1.7 \%)$ & $33(1.7 \%)$ \\
\hline \multicolumn{4}{|l|}{ Education } \\
\hline$<12$ th grade & 2249 (57.9\%) & 2447 (37.5\%) & $661(34.5 \%)$ \\
\hline 12th grade & $967(24.9 \%)$ & $2388(36.6 \%)$ & $736(38.4 \%)$ \\
\hline$>12$ th grade & $668(17.2 \%)$ & 1697 (25.9\%) & $522(27.2 \%)$ \\
\hline \multicolumn{4}{|l|}{ Race } \\
\hline Black & $621(16.0 \%)$ & $907(13.9 \%)$ & $193(10.1 \%)$ \\
\hline White & $3242(83.5 \%)$ & $5553(85.0 \%)$ & 1697 (88.4\%) \\
\hline Others & $21(0.5 \%)$ & $72(1.1 \%)$ & $29(1.5 \%)$ \\
\hline \multicolumn{4}{|c|}{ Family colon cancer history } \\
\hline Yes & $133(3.4 \%)$ & $229(3.5 \%)$ & $127(6.6 \%)$ \\
\hline \multicolumn{4}{|l|}{ Vitamin/mineral use } \\
\hline Yes & $856(22.0 \%)$ & 1451 (22.2\%) & $676(35.2 \%)$ \\
\hline \multicolumn{4}{|l|}{ Alcohol consumption } \\
\hline Baseline: drinkers & $1991(51.3 \%)$ & $2824(43.2 \%)$ & $1165(60.7 \%)$ \\
\hline \multicolumn{4}{|l|}{ Physical activity $\ddagger$} \\
\hline Low & $1376(35.4 \%)$ & $1830(28.0 \%)$ & $517(26.9 \%)$ \\
\hline Medium & $1981(51.0 \%)$ & $3803(58.2 \%)$ & $1151(60.0 \%)$ \\
\hline High & $523(13.5 \%)$ & $894(13.7 \%)$ & $250(13.0 \%)$ \\
\hline
\end{tabular}

* Subjects who reported no tea consumption in both baseline and first follow-up are coded as 'non-tea users'; subjects who consumed tea either at baseline or first follow-up are coded as 'short-term tea users'; subjects who consumed tea at both surveys are considered as 'long-term tea users'.

†Tea consumption information was not available for 21 cases and 2051 non-cases.

$\ddagger$ Physical activity information was not available for 10 subjects. 
Table 2 Tea consumption at baseline (1971-75) and colon cancer risk in the NHEFS Cohort I, by gender

\begin{tabular}{|c|c|c|c|c|c|c|}
\hline \multirow[b]{2}{*}{ Tea consumption } & \multicolumn{2}{|c|}{ Men and women } & \multicolumn{2}{|c|}{ Men only } & \multicolumn{2}{|c|}{ Women only } \\
\hline & $n^{*}$ & $\begin{array}{c}\text { Relative } \\
\text { risk }(95 \% \mathrm{Cl}) \dagger\end{array}$ & $n^{*}$ & $\begin{array}{c}\text { Relative } \\
\text { risk }(95 \% \mathrm{Cl}) \dagger\end{array}$ & $n^{\star}$ & $\begin{array}{c}\text { Relative } \\
\text { risk }(95 \% \mathrm{Cl}) \dagger\end{array}$ \\
\hline \multicolumn{7}{|l|}{ Dichotomous } \\
\hline No & 207 & 1.00 & 105 & 1.00 & 102 & 1.00 \\
\hline Yes & 60 & $0.92(0.67,1.26)$ & 20 & $0.58(0.33,1.01)$ & 40 & $0.98(0.84,1.82)$ \\
\hline \multicolumn{7}{|l|}{ Categorical } \\
\hline Non-user & 207 & 1.00 & 105 & 1.00 & 102 & 1.00 \\
\hline$<1.5$ cups day ${ }^{-1}$ & 31 & $0.98(1.49,0.65)$ & 9 & $0.64(0.30,1.40)$ & 22 & $1.24(0.76,2.04)$ \\
\hline$>1.5$ cups day $^{-1}$ & 29 & $0.85(0.56,1.30)$ & 11 & $0.53(0.26,1.11)$ & 18 & $1.19(0.70,2.03)$ \\
\hline$P$-value for trend & & 0.76 & & 0.15 & & 0.62 \\
\hline
\end{tabular}

* Number of colon cancer cases.

†Adjusted for baseline age, race, education level, BMI, aspirin use, dietary intakes of calories, fat, fibre and calcium, and alcohol use at baseline.

$(P=0.15)$ was observed in men but not women in this study.

The association between tea consumption and colon cancer risk in the NHEFS Cohort II was also examined (Table 3). When data from men and women were combined, individuals reporting tea consumption showed significantly reduced risk for colon cancer compared with non-users (relative risk (RR) $0.58,95 \%$ confidence interval (CI) $0.43,0.78$ ). The association remained significant when tea consumption was examined based on the number of cups of tea consumed each day. In addition, a significant inverse trend $(P<0.01)$ was observed for the association. When men in the NHEFS Cohort II were examined alone, the risk reduction for tea consumption was even greater. A strong dose-response relationship $(P<0.001)$ was observed, with approximately $59 \%$ and $70 \%$ reduced colon cancer risk for those consuming $0-1.5$ cups and for those consuming more than 1.5 cups per day, respectively, compared with men who did not drink tea. Although women who consumed tea demonstrated a reduced relative risk of colon cancer compared with women who did not drink tea, the association did not reach statistical significance $(\mathrm{RR}=0.69,95 \% \mathrm{CI}=0.46$, 1.04).

\section{Discussion}

Findings from epidemiological studies of the relationship between tea consumption and colon cancer risk have been inconsistent. These studies have found tea to be either preventive ${ }^{19}$, a risk factor ${ }^{20}$, or to have no association with colon cancer ${ }^{21}$. The current study found a significantly reduced risk for habitual tea consumers when compared with non-users in the NHEFS Cohort II. In the male population in the study, the inverse doseresponse relationship was significant. The risk reduction measured in the NHEFS 1982-84 survey reached as high as $70 \%$ for men who consumed more than 1.5 cups of tea each day. The relationship for women in the NHEFS Cohort II and for the entire NHEFS Cohort I did not reach statistical significance.

The NHEFS has some powerful advantages as a cohort. Since it reflects the underlying diversity of the US population as a whole, it has greater power to detect an effect than a more homogeneous cohort. In addition, the results from this cohort have great external validity to the US population. Concerns about recall bias are excluded in a cohort design as dietary data are collected prior to the disease diagnosis. The relatively large sample size and the

Table 3 Tea consumption at baseline (1971-75) and colon cancer risk in the NHEFS Cohort II, by gender

\begin{tabular}{|c|c|c|c|c|c|c|}
\hline \multirow[b]{2}{*}{ Tea consumption } & \multicolumn{2}{|c|}{ Men and women } & \multicolumn{2}{|c|}{ Men only } & \multicolumn{2}{|c|}{ Women only } \\
\hline & $n^{*}$ & $\begin{array}{c}\text { Relative } \\
\text { risk }(95 \% \mathrm{Cl}) \dagger\end{array}$ & $n^{\star}$ & $\begin{array}{c}\text { Relative } \\
\text { risk }(95 \% \mathrm{Cl}) \dagger\end{array}$ & $n^{*}$ & $\begin{array}{c}\text { Relative } \\
\text { risk }(95 \% \mathrm{Cl}) \dagger\end{array}$ \\
\hline \multicolumn{7}{|l|}{ Dichotomous } \\
\hline No & 134 & 1.00 & 64 & 1.00 & 70 & 1.00 \\
\hline Yes & 116 & $0.58(0.43,0.78)$ & 52 & $0.40(0.25,0.63)$ & 64 & $0.69(0.46,1.04)$ \\
\hline \multicolumn{7}{|l|}{ Categorical } \\
\hline Non-user & 207 & 1.00 & 64 & 1.00 & 70 & 1.00 \\
\hline$<1.5$ cups day $^{-1}$ & 94 & $0.57(0.42,0.78)$ & 47 & $0.41(0.25,0.66)$ & 47 & $0.67(0.43,1.04)$ \\
\hline$>1.5$ cups day $^{-1}$ & 22 & $0.59(0.35,1.00)$ & 5 & $0.30(0.09,0.98)$ & 17 & $0.74(0.40,1.39)$ \\
\hline$P$-value for trend & & $<0.01$ & & $<0.001$ & & 0.20 \\
\hline
\end{tabular}

* Number of colon cancer cases.

† Adjusted for baseline age, race, education level, BMI, aspirin use, dietary intakes of calories, fat, fibre and calcium, and alcohol use at baseline. 
successful follow-up rate of approximately 90\% provide an excellent data source for the hypotheses in question.

However, there are some limitations to the use of the NHEFS data. The questionnaire at baseline asked whether subjects drank tea or coffee in one question. Thus it is unclear whether subjects who had a positive answer to this question were tea drinkers, coffee drinkers or drinkers of both coffee and tea. Besides, no specific information was obtained regarding either the frequency or the portion of the beverage consumed. In order to avoid misclassification bias, the information was cross-checked with the 24-hour dietary recall data. If an individual reported drinking any coffee but not tea on the particular day of recall, the subject was then categorised as a non-user of tea. If an individual was determined as a tea user in the question but no specific amount of consumption was given in the recall data, the average daily consumption amount among tea users was assigned to that subject. These two approaches to code tea consumption data at baseline aim to minimise possible differential misclassification. Twenty-four hour recall is a valuable tool for capturing frequent (daily) tea consumption but is less reliable in appropriately characterising infrequent consumers. Therefore, the analyses of baseline tea consumption in this study may not capture those who consumed both tea and coffee infrequently in the baseline period, or even tea drinkers who happened not to drink tea at the particular day of the 24-hour dietary recall assessment. As a result, the estimates for the relationship between tea and colon cancer may be biased towards the null and thus weaken the observed associations.

On the other hand, tea consumption in the NHEFS Cohort II was assessed using a semi-quantitative foodfrequency questionnaire (FFQ) for average dietary intakes over the past year. Tea consumption and coffee consumption were queried in separate questions and the frequency, as well as portion size, were assessed separately. The FFQ provides a better quantitative estimate for habitual tea consumption than the 24-hour dietary recall used in the NHEFS Cohort I. The results of this study not only reflect the ability of the FFQ to quantify the habitual tea consumption, but also demonstrate a consistent and stronger preventive effect of tea consumption on colon cancer compared with findings from Cohort I. This is a secondary data analysis study. It is impossible for us to assess the validity of tea consumption data. There is no way to compare the findings from Cohort I and Cohort II to determine which one is more valid. It is, however, clear that the method used to assess tea consumption in Cohort II is a better instrument to capture habitual dietary intake $22-25$. Besides, findings from both cohorts show an inverse relationship between tea consumption and colon cancer risk. In addition, categorical analysis based on tea consumption pattern over the two-survey period, as ordered in Table 1 , suggests that there are $86 \%(P=0.04)$ and $42 \%(P=0.15)$ increased risk for men and women, respectively, when comparing subjects who never drank tea with long-term tea drinkers (data not shown). This finding indirectly supports that the Cohort II results may be more representative of the effect of tea consumption on colon cancer risk.

Data from laboratory studies suggest that specific types of tea have different impacts on carcinogenesis ${ }^{26,27}$. Unfortunately, neither cohort of the NHEFS assessed various types of tea. Lacking information regarding the types of tea used, the results of this study might be prone to misclassification. Given that even the NHANES III (1988-94) dietary assessment data show minimal green tea consumption in the US population, it is reasonable to assume that the majority of the population in the USA consumed black tea at the time that the NHEFS surveys were conducted in the early 1970s and again in the early 1980s. Therefore, the degree of misclassification for types of tea consumed should be minimal. The results seen in this study should be mostly from the effect of black tea.

It is not clear why the preventive effect of tea consumption is stronger in men than women in this study. One explanation may be that the women in this study are significantly younger than are the men. Besides, the risk of colon cancer is lower for women than for men to start with $^{28}$. The non-significant result observed in women may simply be the result of the lower susceptibility to colon cancer risk among this particular population. On the other hand, this study found that the percentage of alcohol users is higher in women than in men in both cohorts. This finding is contradictory to the finding from another US study ${ }^{29}$. It is possible that men in this study may be more likely to underreport alcohol drinking than women $^{30}$. Since alcohol drinking has been linked to colon cancer risk ${ }^{31-34}$, insufficient control for alcohol use may bias the study results. The finding of a higher percentage of women reporting drinking alcohol is consistent across both baseline and first follow-up surveys. The finding of a stronger effect of tea on colon cancer in Cohort II should not be attributed as the result of residual confounding due to insufficient controlling of the self-reported alcohol consumption information.

This study excluded subjects who developed cancer before the study for each of the cohorts. However, Cohort II subjects may have modified their lifestyle and health behaviour simply because they are enrolled in the study. Given the lengthy latent period for colon carcinogenesis, the information collected at the first follow-up survey used as 'baseline' data for Cohort II may have reflected the modified behaviour, especially after close to 10 years in the study since they entered the study in the early 1970 s. Fortunately, NHEFS did not actively contact study participants between each survey, as did another prospective cohort study ${ }^{35}$. Subjects did not receive updated health information from the NCHS. Besides, tea consumption was not considered beneficial during the survey period in the 1970 s and early '80s. Subjects were 
not likely to modify their health behaviour towards tea drinking by simply being in the follow-up study. The direct effect from participation should be minimal. However, residual confounding effects from other lifestyle factors may exist.

In conclusion, this study found a significant inverse association between habitual tea consumption and colon cancer risk among men. The association is less strong in women. Infrequent tea consumption based on a single 24-hour dietary recall with a longer follow-up period did not show significant association. Although the study results should be interpreted cautiously given the nature of the study limitations, an apparent colon cancer prevention effect was seen for men who consumed more than 1.5 cups of tea per day. Considering the increased popularity of the consumption of both green and black tea, this study, along with experimental data, suggests that the preventive effect of tea on colon cancer merits further study.

\section{Acknowledgements}

We are indebted to Soowan Kim for her efforts in constructing the tea variables from both survey rounds and to Christine Cox at the National Center for Health Statistics (NCHS) for clarifying information regarding the NHEFS. We also thank Walter Davis at the Institute for Research in Social Science at the University of North Carolina-Chapel Hill (NC-CH) for co-ordination and preparation of raw data, and Carry Croghan and Tim Goodwin of the Epidemiology Department at UNC-CH for their suggestion for programming. Ellen Shanahan's editorial contributions to the final manuscript are also greatly appreciated.

\section{References}

1 American Cancer Society. Cancer Facts and Figures: 1997. Atlanta, GA: American Cancer Society, Inc., 1997.

2 Silverberg E, Boring C, Squires T. Cancer statistics. CA: a Cancer Journal for Clinicians 1990; 40: 9-26.

3 American Cancer Society. Estimated New Cancer Cases and Deaths by Sex for All Sites, United States, 2000. Atlanta, GA: American Cancer Society, Inc., 2000.

4 Cassidy A, Bingham SA, Cummings JH. Starch intake and colorectal cancer risk: an international comparison. $\mathrm{Br}$. J. Cancer 1994; 69(5): 937-42.

5 Haenszel W, Berg JW, Segi M, Kurihara M, Locke FB. Largebowel cancer in Hawaiian Japanese. J. Natl. Cancer Inst. 1973; 51(6): 1765-79.

6 Whittemore AS, Wu-Williams AH, Lee M, Zheng S, Gallagher $\mathrm{RP}$, Jiao DA, et al. Diet, physical activity, and colorectal cancer among Chinese in North America and China. J. Natl. Cancer Inst. 1990; 82(11): 915-26.

7 Adlercreutz H, Gorbach SL, Goldin BR, Woods MN, Dwyer JT, Hamalainen E. Estrogen metabolism and excretion in Oriental and Caucasian women. J. Natl. Cancer Inst. 1994; 86(14): 1076-82.

8 American Association for Cancer Research. Tea Affects the Formation of Heterocyclic Amines and Their Metabolism in Male and Female Rats. San Francisco, CA: American Association for Cancer Research, 2000.

9 American Association for Cancer Research. Tea Polyphenols
Inhibit Orthotopic Growth and Metastasis of Human Prostate Tumors in Mice. San Francisco, CA: American Association for Cancer Research, 2000.

10 American Association for Cancer Research. Polyphenolic Extracts from Black Tea and Wine Protect Against Azoxymethane-induced Colon Carcinogenesis in Rats. San Francisco, CA: American Association for Cancer Research, 2000 .

11 American Association for Cancer Research. Effect of Tea and Tea Polyphenols on DNA Adduct Formation of Heterocyclic Amines (HCAS) in F344 Rats and CDF-1 Mice. San Francisco, CA: American Association for Cancer Research, 2000.

12 National Center for Health Statistics (NCHS). National Center for Health Statistics: Plan and Operation of the Health and Nutrition Examination Survey, 1971-1973. Vital and Health Statistics. Rockville, MD: US Department of Health, Education, and Welfare, 1973.

13 National Center for Health Statistics (NCHS). National Center for Health Statistics: Plan and Operation of the NHANES I Epidemiologic Follow-up Study, 1982-1984. Vital and Health Statistics. Rockville, MD: US Department of Health and Human Services, 1987.

14 National Center for Health Statistics (NCHS). National Center for Health Statistics: Plan and Operation of the NHANES I Epidemiologic Follow-up Study, 1986. Vital and Health Statistics. Rockville, MD: US Department of Health and Human Services, 1990.

15 National Center for Health Statistics (NCHS). National Center for Health Statistics: Plan and Operation of the NHANES I Augmentation Survey of Adults 25- 74 years: United States, 1974-1975. Washington, DC: NCHS, 1978.

16 Madans JH, Cox CS, Kleinman JC, Makuc D, Feldman JJ, Finucane FF, et al. 10 years after NHANES I: mortality experience at initial followup, 1982-84. Public Health Rep. 1986; 101(5): 474-81.

17 Madans JH, Kleinman JC, Cox CS, Barbano HE, Feldman JJ, Cohen B, et al. 10 years after NHANES I: report of initial followup, 1982-84. Public Health Rep. 1986; 101(5): $465-73$

18 Cornoni-Huntley J, Barbano HE, Brody JA, Cohen B, Feldman JJ, Kleinman JC, et al. National health and nutrition examination I - epidemiologic follow-up survey. Public Health Rep. 1983; 98(3): 245-51.

19 Ji B, Chow W, Hsing A, McLaughlin J, Dai Q, Gao Y, et al. Green tea consumption and the risk of pancreatic and colorectal cancers. Int. J. Cancer 1997; 70(3): 255-8.

20 Hartman T, Tangrea J, Pietinen P, Malila N, Virtanen M, Taylor $\mathrm{P}$, et al. Tea and coffee consumption and risk of colon and rectal cancer in middle-aged Finnish men. Nutr. Cancer 1998; 31(1): 41-8.

21 Tavani A, Pregnolato A, La Vecchia C, Negri E, Talamini R, Franceschi S. Coffee and tea intake and risk of cancers of the colon and rectum: a study of 3,530 cases and 7,057 controls. Int. J. Cancer 1997; 73(2): 193-7.

22 Persson P, Carlsson S, Grill V, Hagman U, Lundgren A, Ostenson C, et al. Food frequency questionnaire versus 7-day weighed dietary record information on dietary fibre and fat intake in middle-aged Swedish men. Scand. J. Social Med. 1998; 26(1): 75-80.

23 Brants H, Lowik M, Brussaard J, Kistemaker C, Van Erp-Baart A. Food consumption methods. Development, reproducibility and validation of a food frequency questionnaire for vitamin B6. Eur. J. Clin. Nutr. 1997; 51(Suppl. 3): S12-8.

24 Metcalf P, Swinburn B, Scragg R, Dryson E. Reproducibility and validity of a food frequency questionnaire in European and Polynesian New Zealanders. Ethnicity Health 1997; 2(4): 278-308.

25 Grootenhuis P, Westenbrink S, Sie C, de Neeling J, Kok F, Bouter L. A semiquantitative food frequency questionnaire 
for use in epidemiologic research among the elderly: validation by comparison with dietary history. J. Clin. Epidemiol. 1995; 48(7): 859-68.

26 Yang C, Lee M, Chen L, Yang G. Polyphenols as inhibitors of carcinogenesis. Environ. Health Perspect. 1997; 105(Suppl. 4): 971-6.

27 Kohlmeier L, Weterings KG, Steck S, Kok FJ. Tea and cancer prevention: an evaluation of the epidemiologic literature. Nutr. Cancer 1997; 27(1): 1-13.

28 Ries L, Kosary C, Hankey B, Miller B, Clegg L, Edwards B. SEER Cancer Statistics Review, 1973-1976. Bethesda, MD: National Cancer Institute, 1999.

29 Dawson D, Archer L. Gender differences in alcohol consumption: effects of measurement. Br. J. Addict. 1992; 87(1): 119-23.

30 Shillington A, Clapp J. Self-report stability of adolescent substance use: are there differences for gender, ethnicity and age? Drug Alcohol Depend. 2000; 60(1): 19-27.
31 Bujanda L. The effects of alcohol consumption upon the gastrointestinal tract. Am. J. Gastroenterol. 2000; 95(12): 3374-82.

32 Hsing A, McLaughlin J, Chow W, Schuman L, Co Chien H, Gridley $\mathrm{G}$, et al. Risk factors for colorectal cancer in a prospective study among US white men. Int.J. Cancer 1998; 77(4): 549-53.

33 Tavani A, Ferraroni M, Mezzetti M, Franceschi S, Lo Re A, La Vecchia C. Alcohol intake and risk of cancers of the colon and rectum. Nutr. Cancer 1998; 30(3): 213-9.

34 Le Marchand L, Wilkens L, Kolonel L, Hankin J, Lyu L. Associations of sedentary lifestyle, obesity, smoking, alcohol use, and diabetes with the risk of colorectal cancer. Cancer Res. 1997; 57(21): 4787-94.

35 Giovannucci E, Colditz G, Stampfer M, Hunter D, Rosner B, Willett W, et al. A prospective study of cigarette smoking and risk of colorectal adenoma and colorectal cancer in US women. J. Natl. Cancer Inst. 1994; 86(3): 192-9. 